\title{
Assurances sociales en Suisse
}

Linus Cavegn, Andy Bühler

Correspondance:

FMH Treuhand Services

Succursale de Bâle

Linus Cavegn/Andy Bühler

Hirzbodenweg 103

CH-4020 Bâle

Tél. 0613195121

Fax 0613195252

linus.cavegn@fmhtreuhand.ch

\section{Modifications importantes entrées en vigueur le $\mathbf{1}^{\mathrm{er}}$ janvier 2006}

En 2006, aucune modification n'entrera en vigueur s'agissant de l'obligation de cotiser à l'AVS/AC/AI/APG. Vous pourrez prendre connaissance des chiffres actuels et historiques en consultant dans le tableau 1.

Nouveau: les personnes exerçant une activité lucrative nées à partir de 1988 ont désormais l'obligation de cotiser.

Par rapport à l'année précédente, les rentes AVS demeurent également inchangées. Les hommes nés en 1941 et les femmes nées en 1942 peuvent faire valoir le droit à la retraite ordinaire.

S'agissant de la LPP (minimum légal) les indices prescrits légalement restent inchangés.

Cela est également valable pour les montants limites exonérés d'impôts pour l'épargne dans le cadre du pilier 3a pour les salariés comme pour les indépendants.

\section{Nouveau numéro AVS}

Le numéro AVS à 11 chiffres valable actuellement sera remplacé par un numéro à 13 chiffres. Il est prévu d'introduire progressivement le nouveau numéro à partir du $1^{\text {er }}$ juillet 2007 . Tous les anciens assurés recevront également un nouveau numéro à 13 chiffres. L'ancien numéro sera asso- cié dans le registre AVS existant au nouveau numéro, de sorte à éviter toute perte de données lors du changement.

Le système de numéro actuel à 11 chiffres se heurte à des limites tant en ce qui concerne le codage utilisé que l'attribution d'un numéro univoque. Le numéro actuel contient des indications codées concernant les assurés comme le jour, le mois et l'année de naissance, le sexe et le groupe des premières lettres du nom de famille et des indications quant à savoir si la personne est suisse ou étrangère. Cet état de fait contrevient aujourd'hui aux exigences de la protection des données.

Le nouveau numéro AVS à 13 chiffres est un numéro totalement anonyme, qui ne permet de tirer aucune conclusion sur l'identité de l'assuré. Il est attribué de manière univoque à une personne et, contrairement à ce qui était le cas pour l'ancien numéro, ne se modifie pas même en cas de changement de nom suite à un mariage. Ainsi, le numéro accompagne l'assuré durant toute sa vie.

La carte AVS grise actuelle est remplacée par une attestation simplifiée. Cette carte contient le numéro AVS, le nom, le prénom et la date de naissance de l'assuré. 


\section{Tableau 1}

Chiffres de référence importants (tous les montants sont en CHF et sont calculés sur une base annuelle)

\begin{tabular}{|c|c|c|c|c|c|c|c|}
\hline & 2000 & 2001 & 2002 & 2003 & 2004 & 2005 & 2006 \\
\hline \multicolumn{8}{|l|}{ AVS / AC / Al / APG } \\
\hline \multicolumn{8}{|l|}{ Cotisations (employeur et salarié, chacun $50 \%$ ) } \\
\hline AVS / Al / APG & $10,10 \%$ & $10,10 \%$ & $10,10 \%$ & $10,10 \%$ & $10,10 \%$ & $10,10 \%$ & $10,10 \%$ \\
\hline moins de 106800 & $3,00 \%$ & $3,00 \%$ & $3,00 \%$ & $2,50 \%$ & $2,00 \%$ & $2,00 \%$ & $2,00 \%$ \\
\hline $106801-267000$ & $2,00 \%$ & $2,00 \%$ & $2,00 \%$ & $1,00 \%$ & $0,00 \%$ & $0,00 \%$ & $0,00 \%$ \\
\hline $\begin{array}{l}\text { Cotisation minimale à I'AVS pour les personnes } \\
\text { sans activité lucrative }\end{array}$ & 390 & 390 & 390 & 425 & 425 & 425 & 425 \\
\hline Début de l'obligation de cotiser pour salariés à partir de l'année & 1982 & 1983 & 1984 & 1985 & 1986 & 1987 & 1988 \\
\hline
\end{tabular}

de naissance (à partir du 1er janvier)

\section{Montants limites et franchises}

Franchise pour les retraités (au-delà uniquement obligation de cotiser à l'AVS, mais pas à l'AC) (avec déclaration de renonciation)*

(possible uniquement si une activité lucrative principale est exercée)

* La caisse de compensation évalue ce qui est réputé activité lucrative accessoire.

\section{Rentes}

Rente AVS simple, rente AI, rente de couple (rente complète selon échelle 44)

\begin{tabular}{|c|c|c|c|c|c|c|c|}
\hline Maximum & 24120 & 24720 & 24720 & 25320 & 25320 & 25800 & 25800 \\
\hline Minimum & 12060 & 12360 & 12360 & 12660 & 12660 & 12900 & 12900 \\
\hline Rente de couple & 36180 & 37080 & 37080 & 37980 & 37980 & 38700 & 38700 \\
\hline \multicolumn{8}{|c|}{ Début du droit au versement d'une rente années de naissance (chaque fois à partir du $1^{\text {er }}$ du mois suivant la date de naissance) } \\
\hline Femmes & 1938 & * & 1939 & 1940 & 1941 & ** & 1942 \\
\hline Hommes & 1935 & 1936 & 1937 & 1938 & 1939 & 1940 & 1941 \\
\hline
\end{tabular}

* Au $1^{\text {er }}$ janvier 2001, l'âge de la retraite AVS a été porté pour les femmes à 63 ans. Les femmes nées en 1939 ont été les premières à être concernées par cette augmentation. Leur droit ordinaire à la retraite naissait en 2002.

** L'âge ordinaire de la retraite pour les femmes a été porté au 1er janvier 2005 à 64 ans.

LPP (minimum légal)

\begin{tabular}{lrrrrrrr}
\hline Revenu annuel à prendre en compte (maximum) & 72360 & 74160 & 74160 & 75960 & 75960 & 77400 & 77400 \\
\hline Déduction de coordination & 24120 & 24720 & 24720 & 25320 & 25320 & $22575^{*}$ & $22575^{*}$ \\
\hline Salaire annuel maximum assuré & 48240 & 49440 & 49440 & 50640 & 50640 & 54825 & 54825 \\
\hline Salaire annuel minimum assuré & 3015 & 3090 & 3090 & 3165 & 3165 & 3225 & 3225 \\
\hline Limite de revenu inférieur & 24120 & 24720 & 24720 & 25320 & 25320 & 19350 & 19350
\end{tabular}

* $7 / 8$ de la rente AVS maximale simple.

Epargne pilier $3 a$

$2^{\mathrm{e}}$ pilier (LPP): maximum (= $8 \%$ du salaire LPP maximal assuré)

Montant limite exonéré d'impôt pour les indépendants sans $2^{\mathrm{e}}$ pilier:

5933

5933

6077

6077

6192

6192

$20 \%$ du revenu mais maximum (= $40 \%$ du salaire LPP maximal assuré

28944

29664

29664

30384

30384

30960

30960 